\title{
Objetivos e desempenho da cooperação interfirmas: efeitos das práticas de gestão da cooperação e do escopo de transação
}

\author{
Jessica Giovana Nolli \\ https://orcid.org/0000-0001-7031-3908 | E-mail:jessicanolli@hotmail.com \\ Ilse Maria Beuren \\ https://orcid.org/0000-0003-4007-6408 | E-mail: ilse.beuren@gmail.com
}

\section{Resumo}

Objetivo: Este estudo analisa o efeito das práticas de gestão da cooperação e do escopo de transação na relação entre os objetivos da cooperação e o desempenho da cooperação em empresas da indústria têxtil. Método: Uma survey foi realizada em empresas da indústria têxtil brasileira, tendo como amostra os 93 gestores respondentes da pesquisa. Para análise das hipóteses aplicou-se a técnica de Modelagem de Equações Estruturais.

Resultados: Os resultados da pesquisa mostram que os respondentes avaliaram a cooperação como estrategicamente importante. Porém, para alcançar os objetivos pretendidos com a aliança estratégica é necessário que as práticas de gestão da cooperação e o escopo de transação estejam alinhados. A relevância estratégica da cooperação impactou positivamente o desempenho da cooperação, quando associada ao compartilhamento de informações, à interação entre os parceiros e às atividades desenvolvidas entre os parceiros da cooperação.

Contribuições: O estudo contribui para a literatura e a prática das empresas ao associar construtos da cooperação voltados à estratégia para a competitividade com o desempenho da cooperação interfirmas. Os resultados revelaram que quanto maior a interação dos envolvidos, o compartilhamento de informações e as atividades desenvolvidas na cooperação interfirmas maior será a associação entre os objetivos e o desempenho da cooperação.

Palavras-chave: Objetivos da cooperação. Práticas de gestão da cooperação. Escopo de transação. Desempenho da cooperação interfirmas. 


\section{Introdução}

A Teoria da Cooperação, originada de perspectivas sociais e econômicas, tem como premissa que os objetivos podem ser alcançados por todos ou quase todos os parceiros envolvidos na cooperação (May \& Doob, 1937). A cooperação interfirmas tem sido uma estratégia adotada de forma crescente nas últimas décadas nas organizações para obterem vantagens competitivas (Dekker, Sakaguchi \& Kawai, 2013; Ding, Dekker \& Groot, 2013; Anderson \& Dekker, 2014; Dekker, 2016; Dekker, Ding \& Groot, 2016). No entanto, a cooperação não ocorre apenas pela existência de uma atividade realizada conjuntamente, mas pela dinamicidade das interações inseridas nesse ambiente (Das \& Teng, 1998).

Diversos tipos de relacionamentos interfirmas são abordados na literatura, tais como: cadeia de suprimentos (Mouritsen, Hansen \& Hansen, 2001), terceirização (Langfield-Smith \& Smith, 2003), franquia (Wu, 2015), acordo de produção conjunta (Eiriz, 2001), joint venture (Groot \& Merchant, 2000). Algumas empresas terceirizam atividades não primordiais, como treinamento e benefícios de funcionários, outras fazem acordos cooperativos com organizações parceiras (Groot \& Merchant, 2000). As relações interfirmas podem permitir a inserção de ações para aumentar o processo de aprendizagem e os incentivos para as inovações de processos e produtos no contexto das organizações parceiras (Dias, 2018).

A cooperação é relevante nos relacionamentos interfirmas (Das \& Teng, 1998). Todavia, ela requer que as organizações parceiras estejam alinhadas à estratégia colaborativa definida, o que implica planejar métricas para aferir o quanto dessa estratégia está sendo alcançada. A comunicação deve servir como apoio para disseminar uma visão comum e incentivar os parceiros a aprimorarem as relações e os resultados da cooperação. O envolvimento dos parceiros permite reportar dificuldades e eventuais disputas, além de compartilhar informações e adequar a estratégia para acompanhar a evolução das condições das organizações (Kaplan, Norton \& Rugelsjoen, 2010).

O envolvimento de diversas atividades organizacionais na cooperação requer maior comprometimento dos parceiros com a aliança (Kalaignanam, Shankar \& Varadarajan, 2007), para proporcionar benefícios estratégicos às empresas parceiras. Nesta perspectiva, Contractor e Lorange (1988) e Groot e Merchant (2000) investigaram as razões estratégicas para as organizações se envolverem em cooperação interfirmas. Mahama (2006) pesquisou a importância do compartilhamento de informações entre as empresas parceiras. Dekker et al. (2013) analisaram a interação entre funcionários no processo de cooperação.

Alguns estudos relataram alta taxa de insucesso na cooperação interfirmas, devido aos riscos envolvidos nessas transações (Lunnan \& Haugland, 2008). Redes verticais de cooperação interfirmas podem fazer com que pequenas empresas executem atividades intermediárias e não sejam beneficiadas apropriadamente na cooperação, resultando em relações superficiais entre as empresas parceiras e a inexistência de mecanismos coordenados de controle (Dias, 2018). No entanto, diferentes tipos de relacionamentos interfirmas podem estimular a concorrência, principalmente em setores mais fragmentados, além de que poucas empresas conseguem alcançar sucesso sem parcerias (Groot \& Merchant, 2000).

Para que as organizações alcancem seus objetivos, é necessário que os interesses dos parceiros estejam alinhados. Nesta perspectiva, Dekker et al. (2016) examinaram a influência dos objetivos da cooperação nas práticas de gestão e no desempenho das relações interfirmas sob a perspectiva dos profissionais de administração financeira cadastrados no Controllers Institute da Holanda. Pautados nos resultados da pesquisa, os autores sugerem que estudos futuros analisem como o alinhamento entre os objetivos da cooperação, o escopo de transação e as práticas de cooperação afetam o desempenho organizacional. 
Dado o exposto, tem-se a seguinte pergunta de pesquisa: Qual o efeito das práticas de gestão da cooperação e do escopo de transação na relação entre os objetivos da cooperação e o desempenho da cooperação interfirmas? As operações interfirmas podem ser encontradas em diversas indústrias, mas algumas possuem um direcionamento mais forte para a formação de alianças devido à natureza das atividades que desenvolvem, como no caso da indústria têxtil, alvo deste estudo, cuja cadeia produtiva abrange a produção de fios e filamentos, manufaturados têxteis, estampagem (La Rovere, Hasenclever \& Melo, 2001) e compreende os segmentos de fiação, tecelagem e acabamento (Dias, 2018).

Gibbon (2002) destaca que, desde a década de 1990, as empresas industriais têxteis vêm passando por transformações e a maioria delas buscou alternativas como inovações técnicas, melhorias na qualidade e parcerias estratégicas (cadeia de suprimentos, terceirização, franquias, acordos de produção conjunta e joint ventures) para ampliar as trocas de informações e aumentar a eficiência. Assim, o objetivo geral do estudo é analisar o efeito das práticas de gestão da cooperação e do escopo de transação na relação entre os objetivos da cooperação e o desempenho da cooperação em empresas da indústria têxtil.

Estudos teórico-empíricos têm focalizado essas relações e seus impactos nas relações interfirmas (Dekker et al., 2016). Ding et al. (2013) observaram que, quando há risco maior no âmbito das relações interfirmas, as empresas tornam-se mais criteriosas na seleção dos seus parceiros. Dekker et al. (2016) constataram que as características das transações são determinadas pela relevância estratégica da cooperação, o que influencia as práticas de gestão de desempenho das organizações. Porém, esses estudos não investigaram a influência dos objetivos e práticas de gestão da cooperação e do escopo de transação no desempenho da cooperação interfirmas (Dekker et al., 2016).

As pesquisas brasileiras sobre cooperação interfirmas estão crescendo em virtude da proximidade entre a literatura e o cotidiano das organizações no que tange aos impactos da cooperação estabelecida pelos parceiros (Balestrin, Verschoore \& Reyes Jr, 2010). Nas últimas décadas, o papel da contabilidade na gestão das relações interfirmas passou a ser um tema de interesse dos pesquisadores, cuja atenção coincidiu com o aumento significativo das relações de cooperação interfirmas (Anderson \& Dekker, 2014). Assim, com este estudo, busca-se ampliar a literatura sobre as formulações estratégicas da cooperação e o impacto dessas relações no desempenho das organizações.

A contribuição teórica do estudo está em fornecer evidências das práticas de gestão da cooperação e do escopo de transação como mediadores da relação entre os objetivos e desempenho da cooperação. Contribui ao demonstrar que o alinhamento entre os objetivos e as práticas de gestão da cooperação interfirmas, assim como o escopo de transação, tendem a resultar em melhor desempenho dessas relações (Dekker et al., 2016). Também possui contribuição prática, por avaliar os efeitos desses fatores na cooperação interfirmas entre empresas da indústria têxtil, que são determinantes para sua competitividade e sobrevivência, devido ao impacto superior esperado no desempenho organizacional dos parceiros em relação ao desempenho das empresas não cooperadas (Centenaro \& Laimer, 2017).

O restante do trabalho está estruturado como segue: na segunda seção, discorre-se sobre a associação dos objetivos da cooperação com o desempenho da cooperação e o efeito mediador das práticas de gestão e do escopo da transação nesta relação; na terceira, apresenta-se a metodologia da pesquisa; na quarta, são evidenciados os resultados da pesquisa empírica e a discussão dos resultados; e, na última seção, são apresentadas as considerações finais. 


\section{Base teórica e hipóteses}

\subsection{Objetivos da cooperação e desempenho da cooperação}

A Teoria da Cooperação, criada por May e Doob (1937), aduz que os indivíduos cooperam entre si quando se dedicam para atingir objetivos semelhantes ou complementares que podem ser compartilhados e alcançam desempenho superior quando o objetivo é alcançado na mesma proporção pelos parceiros. A cooperação é definida por Das e Teng (1998) como sendo a intenção de uma organização parceira de alcançar interesses mútuos e compatíveis com outras organizações em vez de atuarem de maneira oportunista.

Os objetivos da cooperação referem-se aos motivos estratégicos que levam as empresas a participarem dos relacionamentos de cooperação interfirmas (Dekker et al., 2016). De acordo com Groot e Merchant (2000), os principais objetivos da cooperação são: redução de custos (acesso a mão de obra relativamente barata); acesso a mercados (superar barreiras comerciais); desenvolvimento tecnológico; e redução de riscos.

Quando os objetivos da cooperação e individuais divergem entre as empresas parceiras há conflitos de interesses, isso exige o envolvimento maior dos funcionários para lidar com esses conflitos (Kaplan et al., 2010). As empresas muitas vezes buscam objetivos múltiplos, o que aumenta a relevância estratégica e a complexidade de controlar a cooperação (Reuer \& Ariño, 2007). Se prevalecerem objetivos individuais, isso influenciará a cooperação realizada (Dekker et al., 2016).

Nesse contexto, o sistema de controle gerencial deve fomentar o equilíbrio entre os interesses e as decisões a serem tomadas em relação aos objetivos e as estratégias da cooperação interfirmas (Kaplan et al., 2010). A função facilitadora do sistema está na tomada de decisões consistentes com a estratégia organizacional (Grafton, Lillis \& Widener, 2010). Portanto, ele tem a função de facilitar a tomada de decisões para alcançar os objetivos colaborativos, além de conciliar os objetivos divergentes entre as empresas parceiras (Ireland, Hitt \& Vaidyanath, 2002).

Isso sugere que as alianças estratégicas precisam ser avaliadas pelo seu desempenho. Embora estudos anteriores tenham investigado o desempenho da cooperação, não se observa consenso entre os pesquisadores sobre o uso de medidas de desempenho da cooperação (Das \& Teng, 2003). Alguns pesquisadores propõem medidas subjetivas, como a satisfação percebida ou expectativa alcançada com a cooperação (Mahama, 2006). Outros utilizam medidas objetivas, como lucro, custos, receitas (Contractor \& Lorange, 1998). Destaca-se que a relação interfirmas pode ser destinada não apenas para fins econômicofinanceiros, como geração de lucros, mas também para facilitar a troca de conhecimento e de informações entre as empresas parceiras (Kogut, 1988).

A cooperação envolve uma abordagem de mensuração mais equilibrada e subjetiva da promessa do que pode ser alcançado (Anderson, 1990). Utilizar métricas mais formais de avaliação de desempenho da cooperação pode provocar o rompimento antecipado da relação devido à falta de tempo necessário para a cooperação demonstrar seu valor. Portanto, as medidas de percepção da satisfação dos parceiros com o desempenho da cooperação fornecem informações mais amplas sobre o pretendido com a cooperação (Dekker, 2016). Neste sentido, formula-se a primeira hipótese da pesquisa:

\section{- $H_{1}$ : Há relação positiva entre os objetivos da cooperação e o desempenho da cooperação.}

Quando a relevância dos objetivos da cooperação aumenta nas organizações, aumenta também a coordenação e controle das práticas de cooperação (Dekker et al., 2016). Para esses autores, quando uma empresa almeja múltiplos objetivos da cooperação, relevantes estrategicamente, isso influencia no tipo de ativos utilizados no relacionamento, na interação dos expansores de fronteiras (boundary spanners), no escopo de transação, nas práticas de gestão e nas atividades de cooperação. 


\subsection{Objetivos, práticas de gestão e desempenho da cooperação}

As práticas de gestão da cooperação abrangem a interação dos expansores de fronteiras (boundary spanners) e o compartilhamento de informações (Mahama, 2006), os quais são determinantes para os objetivos da cooperação e espera-se que impactem o desempenho da relação interfirmas (Dekker et al., 2016). As práticas de gestão da cooperação são necessárias para gerenciar a relação interfirmas, por exemplo, planejamento, definição de metas de desempenho, interação entre os parceiros, suporte aos envolvidos, resolução conjunta de problemas, compartilhamento de informações e acompanhamento periódico (Dekker et al., 2013).

A interação dos expansores de fronteiras (boundary spanners) favorece as empresas trabalharem conjuntamente e monitorarem o comportamento e o desempenho do parceiro na cooperação (Ittner, Larcker, Randall \& Rajan, 1999). Essa interação é realizada por indivíduos responsáveis pela gestão interfirmas e que interagem frequentemente com as empresas parceiras (Wilson \& Barbat, 2015). Os indivíduos dessa interação são os gestores designados formalmente para controlar as alianças, os gestores da cadeia de suprimentos e os envolvidos nas relações comerciais das empresas que se unem para trocar informações relativas à cooperação (Wilson \& Barbat, 2015).

O compartilhamento de informações implica a troca de informações relevantes e particulares entre os parceiros (Mahama, 2006). Compartilhar informações é primordial para reduzir a assimetria da informação e incentivar a união dos esforços colaborativos entre os parceiros (Corsten, Gruen \& Peyinghaus, 2011). Mesmo que as práticas de cooperação possam ser realizadas em diferentes etapas do relacionamento interfirmas, elas se sobrepõem e são interdependentes para reforçar a eficácia da relação.

As práticas de gestão da cooperação são complementares, de modo que o uso condicionado de uma prática associa-se ao maior uso de outra (Mahama, 2006). Assim, quando uma cooperação é considerada estrategicamente relevante, ela estimula as empresas a impulsionarem as atividades de mensuração do desempenho e isto estará associado ao maior compartilhamento de informações e interação dos expansores de fronteiras (boundary spanners) (Mahama, 2006). Quando aumenta a relevância estratégica do risco da cooperação e da transação, as práticas se complementam, gerando mais valor para as organizações parceiras (Mahama, 2006; Dekker et al., 2013).

Dekker et al. (2016) defendem que o aumento da importância estratégica da cooperação resulta em maior interação dos expansores de fronteiras (boundary spanners) e exige maior compartilhamento de informações entre os parceiros para realizar mais atividades em conjunto, de modo a alinhar os recursos e beneficiar os parceiros. O desempenho da cooperação é melhorado com a compreensão dos objetivos e atividades da aliança estratégica, visto que o compartilhamento de informações permite corrigir ações, reduzir incertezas, promover aprendizagem, alinhar as expectativas das empresas e expandir a interação entre os responsáveis pela cooperação (Mahama, 2006). Desse modo, quando uma empresa visa atingir seus objetivos da cooperação, isso impactará as práticas de gestão da cooperação e, consequentemente, no desempenho dessa relação (Dekker et al., 2016).

Os objetivos da cooperação são projetados para melhorar o desempenho das empresas (Mahama, 2006), sendo que o compartilhamento de informações e a interação dos expansores de fronteiras (boundary spanners) medeiam essa interferência, pois, quando as empresas parceiras almejam objetivos múltiplos, isso provoca maior exposição a diferentes fatores internos, podendo interferir nas atividades organizacionais e, consequentemente, na cooperação, o que exige dos gestores maior controle, acompanhamento e comunicação entre os parceiros (Dekker et al., 2016). A mediação é esperada pela importância do alinhamento entre os objetivos e as práticas de gestão da cooperação, com vistas no desempenho da cooperação (Mahama, 2006). Assim, formula-se a segunda hipótese:

- $H_{2}$ : Há relação positiva entre os objetivos da cooperação e o desempenho da cooperação, mediada pelas práticas de gestão da cooperação. 
Além das práticas de gestão da cooperação, outro fator que pode influenciar a relação entre os objetivos e o desempenho da cooperação é a extensão com que as atividades dos parceiros são sobrepostas e auxiliam no conhecimento, o escopo de transação (Khanna, Gulati \& Nohria, 1998).

\subsection{Objetivos da cooperação, escopo de transação e desempenho da cooperação}

O escopo de transação é definido como a amplitude das atividades desenvolvidas nas organizações, como pesquisa e desenvolvimento, marketing e produção, que elas concordam em realizar nas relações de cooperação interfirmas (Varadarajan \& Cunningham, 1995). Para Oxley e Sampson (2004), o escopo de transação refere-se à combinação de múltiplas atividades da cadeia de valor em uma cooperação. O escopo de transação pode ser utilizado como uma proxy para o valor comercial da cooperação, pois os investidores a utilizam para estimar o fluxo de receita futura das empresas parceiras (Kalaignanam et al., 2007).

Este escopo pode influenciar na alteração de valores das empresas parceiras (Varadarajan \& Cunningham, 1995). As cooperações com maior escopo de transação são passíveis de gerar receitas e ganhos maiores do que cooperações com escopo mais restritivo. Desse modo, uma cooperação que engloba diversos setores fornece indícios de maior potencial financeiro do que uma que abrange apenas alguns setores (Kalaignanam et al., 2007).

Um amplo escopo de transação pode indicar maior comprometimento dos parceiros com a cooperação do que um escopo de transação menor (Kalaignanam et al., 2007). Espera-se que as organizações com escopo de transação maior beneficiem-se de forma mais ampla do que as que possuem um escopo menor, pois uma cooperação com um escopo de transação maior propicia mais oportunidades de ganhos do que um escopo menor, que limita a capacidade de lucros (Li, Tang, Okano \& Gao, 2013). Entretanto, relações de cooperação com escopo de transação maior são mais complexas de administrar, implica gerenciar questões de coordenação e cooperação das atividades realizadas na cooperação para impactar, positivamente, o desempenho (Dekker, Donada, Mothe \& Nogatchewsky, 2019).

Desse modo, as empresas escolhem mecanismos para alcançar seus objetivos da cooperação e o escopo de transação pode mediar essa relação. $O$ efeito da mediação é esperado porque o escopo de transação retrata o ambiente interno da cooperação, os setores envolvidos que influenciam no alinhamento entre os objetivos e o alcance do desempenho das empresas parceiras (Dekker et al., 2016). Com base no exposto, formula-se a terceira hipótese da pesquisa:

- $\mathrm{H}_{3}$ : Há relação positiva entre os objetivos da cooperação e o desempenho da cooperação, mediada pelo escopo de transação.

O escopo de transação é uma característica importante da cooperação e estabelece a estrutura (Mishra, Chandrasekaran \& Maccormack, 2015) e os desafios de gestão da cooperação (Dekker et al., 2016). A cooperação com escopo maior tende a propiciar aos parceiros maiores benefícios, porém os expõe a maiores riscos e interdependências, que precisam ser gerenciados (Dekker et al., 2019). Assim, espera-se que os objetivos e as práticas de gestão da cooperação estejam inter-relacionados (Dekker et al., 2016), e que impactem o desempenho da cooperação (Mahama, 2006).

$\mathrm{Na}$ Figura 1, apresenta-se o modelo teórico da pesquisa, com destaque para as hipóteses propostas neste estudo. 


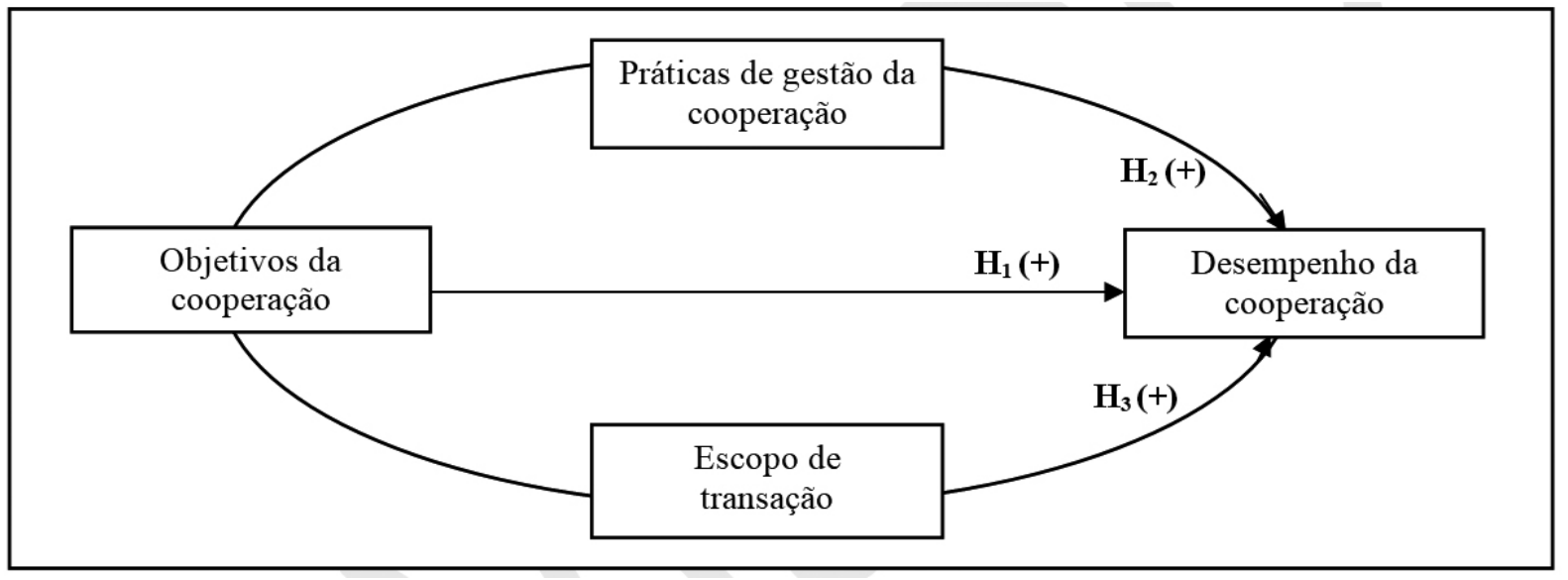

Figura 1. Modelo teórico da pesquisa

Fonte: elaboração própria.

Observam-se na Figura 1 as relações propostas para as três hipóteses da pesquisa. Em consonância com a revisão da literatura apresentada, espera-se relação positiva nas três hipóteses formuladas.

\section{Metodologia da pesquisa}

Uma pesquisa de natureza descritiva foi realizada a partir de um levantamento em empresas da indústria têxtil brasileira. A população da pesquisa compreendeu as empresas brasileiras exportadoras e importadoras listadas no Ministério da Indústria, Comércio Exterior e Serviços (MDIC) e as empresas participantes do Programa de Internacionalização da Indústria Têxtil e de Moda Brasileira (Texbrasil), da Associação Brasileira da Indústria Têxtil e de Confecção (ABIT) em parceria com a Agência Brasileira de Promoção de Exportações e Investimentos (Apex-Brasil). A opção por estas empresas deve-se ao seu destaque nacional e por atenderem aos contornos do propósito da pesquisa.

Nessa forma de busca, identificaram-se 1.104 empresas. Dessas, 773 foram localizadas na rede social Linkedin. Após a identificação das empresas, buscaram-se no Linkedin os profissionais que nelas atuam nos cargos de presidente, diretor presidente, Chief Executive Officer (CEO), diretor financeiro, Chief Financial Officer (CFO), gerente, manager, coordenador e controller. Optou-se por esses sujeitos de pesquisa por fazerem parte do processo de gestão e, consequentemente, das transações relativas às atividades de cooperação. Esses também foram os sujeitos de pesquisa do estudo de Dekker et al. (2016), sob o argumento de sua importância no processo de gestão das organizações.

Em seguida, foi enviado o convite de participação da pesquisa para os 1.122 profissionais identificados, no limite de três gestores por empresa. Um total de 465 profissionais aceitou o convite, para os quais foi enviado o link do instrumento de pesquisa pela plataforma Google Forms, e obteve-se 96 questionários respondidos. Desses, 93 foram considerados válidos, tendo em vista que dois respondentes não possuíam a função estabelecida para este estudo, e um respondente assinalou as mesmas opções de respostas, o que sugere falta de qualidade nas respostas.

Faul, Erdfelder, Buchner e Lang (2009) destacam que o tamanho mínimo da amostra pode ser calculado por meio do software $G^{*}$ Power 3.1.9. Neste estudo, um construto (desempenho da cooperação) recebe o maior número de setas, sendo quatro setas. Desse modo, a amostra mínima deve ser formada por 85 respondentes, ao nível de significância de $5 \%$ e tamanho do efeito médio. Ressalta-se que o nível de análise centra-se nas empresas. 
Para avaliar o efeito das práticas de gestão da cooperação interfirmas e do escopo de transação na relação entre os objetivos da cooperação e o desempenho da cooperação, todos os construtos foram mensurados por meio de itens múltiplos, cujas assertivas foram baseadas em estudos anteriores (Apêndice A). Cada assertiva foi fundamentada em escala Likert ou de diferencial semântico de cinco pontos.

Os objetivos da cooperação foram baseados em estudos anteriores que identificaram as razões estratégicas para as empresas envolverem-se em relações interfirmas (Dekker et al., 2016). Os autores basearam esse construto nos estudos de Contractor e Lorange (1988) e Groot e Merchant (2000). Dekker et al. (2016) identificaram 14 objetivos da cooperação, considerados nesta pesquisa.

Quanto ao escopo de transação, as relações de cooperação com escopo maior são mais complexas de controlar, exigem maior coordenação dos envolvidos (Kalaignanam et al., 2007). Ding et al. (2013) e Dekker et al. (2016) mensuraram o escopo de transação a partir de 8 itens que identificam as atividades de cooperação: pesquisa e desenvolvimento, compras, engenharia, produção, marketing e vendas, serviço pós-venda, troca de conhecimento e prestação de serviço.

As práticas de gestão da cooperação utilizadas neste estudo compreendem o compartilhamento de informações e a interação dos expansores de fronteiras (boundary spanners) e foram baseados nos estudos de Dekker et al. (2016). Para esses autores, o compartilhamento de informações abrange 6 itens, que se referem à extensão com que os parceiros trocam informações sobre: custos, vendas, desenvolvimento de produtos, atividades de marketing, performance operacional e recrutamento e treinamento.

A interação dos expansores de fronteiras (boundary spanners) pode acontecer em diferentes níveis organizacionais e quanto maior for o envolvimento dos colaboradores maior a probabilidade de se alcançar as metas pretendidas (Dekker et al., 2013). Dekker et al. (2016) mensuraram esse construto por meio de 3 itens, questionando a frequência com que os funcionários consultam a alta administração, os gerentes ativos e os participantes da cooperação para discussão dos resultados.

Para o desempenho da cooperação, foi utilizado o instrumento de pesquisa de Dekker et al. (2016). A partir de 20 itens, avaliaram o desempenho da cooperação, combinando medidas financeiras e não financeiras. Questionaram quão relevantes são diferentes dimensões de desempenho, como custos, lucro, fluxo de caixa, fatia de mercado, qualidade dos produtos, satisfação dos clientes, satisfação dos empregados. Pontuação maior indica o uso de um conjunto amplo de medidas para avaliar o desempenho da cooperação.

As tipologias de cooperação interfirmas mais mencionadas na literatura são apontadas com base nas definições de Eiriz (2001) e Meira, Kartalis, Tsamenyi e Cullen, (2010), sendo seis alternativas de seleção. O respondente tinha a opção de selecionar diversos tipos, visto que uma organização pode envolver-se em diferentes formas de cooperação, além da opção de incluir outro tipo de cooperação não mencionado. Essas tipologias foram utilizadas como variáveis de controle para diferenciar os tipos de cooperação e sua influência na cooperação.

Os instrumentos de pesquisa utilizados pelos autores mencionados foram traduzidos do idioma Inglês para o Português e, após, foi realizada a tradução reversa para validação da tradução. O instrumento foi revisado por um pesquisador especialista nesta temática, para validar a tradução e propor eventuais adequações. Para eliminar possíveis incoerências e mitigar dificuldades de entendimento, o questionário foi submetido a três pesquisadores da área e, após suas considerações, realizaram-se alguns ajustes semânticos.

A coleta de dados foi realizada no período de outubro de 2018 a maio de 2019. Aos dados coletados aplicou-se a técnica de Modelagem de Equações Estruturais, por meio do SmartPLS versão 3.0. Para testar as mediações propostas nas hipóteses, utilizou-se o bootstrapping conforme recomendado por Hayes (2009), tendo em vista que esse teste não pressupõe a distribuição normal da amostra e, portanto, é superior ao teste de Sobel. Segundo o autor, o bootstrapping cria uma representação empírica da distribuição do efeito indireto, com reamostragens para reproduzir a população e proporcionar maior robustez aos resultados. 


\section{Análise dos resultados}

\subsection{Caracterização dos respondentes da pesquisa e das empresas}

$\mathrm{Na}$ Tabela 1, apresenta-se o perfil dos respondentes da pesquisa, com destaque para o gênero, faixa etária, escolaridade, formação acadêmica e função/cargo que ocupa na empresa.

Tabela 1

Perfil dos respondentes da pesquisa

\begin{tabular}{|c|c|c|c|c|c|}
\hline Gênero & Qtd. & $\%$ & Faixa Etária & Qtd. & $\%$ \\
\hline Masculino & 76 & $82 \%$ & Menos de 30 anos & 9 & $10 \%$ \\
\hline Feminino & 17 & $18 \%$ & De 31 a 40 anos & 27 & $29 \%$ \\
\hline Total & & & De 41 a 50 anos & 35 & $38 \%$ \\
\hline Escolaridade & Qtd. & $\%$ & De 51 a 60 anos & 15 & $16 \%$ \\
\hline Ensino Médio & 3 & $3 \%$ & De 61 a 70 anos & 7 & $7 \%$ \\
\hline Ensino Superior & 25 & $27 \%$ & Total & 93 & $100 \%$ \\
\hline Especialização ou MBA & 51 & $55 \%$ & Função/Cargo & Qtd. & $\%$ \\
\hline Mestrado ou Doutorado & 14 & $15 \%$ & Diretor & 32 & $35 \%$ \\
\hline Total & 93 & $100 \%$ & Gerente e Coordenador & 56 & $60 \%$ \\
\hline Formação Acadêmica & Qtd. & $\%$ & Controller & 4 & $4 \%$ \\
\hline Negócios & 68 & $73 \%$ & Não declarado & 1 & $1 \%$ \\
\hline Demais áreas & 23 & $25 \%$ & Total & 93 & $100 \%$ \\
\hline Não declarada & 2 & $2 \%$ & & & \\
\hline Total & 93 & $100 \%$ & & & \\
\hline
\end{tabular}

Nota. Negócios (Administração, Ciências Contábeis, Economia). Demais áreas (Licenciatura em Letras, Direito, Tecnologia da Informação, Física, Arquitetura, Química e Engenharia).

Fonte: dados da pesquisa.

Observa-se na Tabela 1 que $82 \%$ dos respondentes são do gênero masculino. A faixa etária dos respondentes concentra-se entre 41 e 50 anos, representando 38\% da amostra, e $29 \%$ têm entre 31 e 40 anos. Em relação ao nível de escolaridade dos respondentes, 27\% possuem nível superior, 55\% possuem pós-graduação na modalidade lato sensu (especialização), 15\% possuem pós-graduação na modalidade stricto sensu (mestrado ou doutorado). Dos três respondentes que indicaram grau de escolaridade ensino médio, dois não declararam formação acadêmica e um apontou formação de tecnólogo, assim foi classificado como demais áreas. Destaca-se que 73\% dos respondentes tem formação na área de negócios.

O cargo ou função com maior quantidade de respondentes da pesquisa foi o de gerente e coordenador, totalizando 56 respondentes, representando $60 \%$ da amostra analisada, seguido de diretor, com 35\%. A média de tempo no cargo ou na função é de 10 anos, o que indica que os respondentes estão, há algum tempo, nessas empresas. O perfil dos respondentes sugere que eles reúnem as condições necessárias para responder as questões do instrumento de pesquisa.

$\mathrm{Na}$ Tabela 2 são apresentadas as principais atividades econômicas das empresas, além da classificação das mesmas pelo porte e mercado de atuação. 
Tabela 2

Segmento de atuação e classificação das empresas

\begin{tabular}{|c|c|c|c|c|c|}
\hline Atividade econômica & Qtd. & $\%$ & Mercado de atuação & Qtd. & $\%$ \\
\hline Indústria & 82 & $88 \%$ & Regional & 2 & $2 \%$ \\
\hline Comércio & 10 & $11 \%$ & Nacional & 29 & $31 \%$ \\
\hline Serviços & 1 & $1 \%$ & Nacional e Internacional & 62 & $67 \%$ \\
\hline Total & 93 & $100 \%$ & Total & 93 & $100 \%$ \\
\hline \multicolumn{4}{|c|}{ Faturamento médio anual } & Qtd. & $\%$ \\
\hline \multicolumn{4}{|c|}{ Menor ou igual a $R \$ 2,4$ milhões } & 10 & $11 \%$ \\
\hline \multicolumn{4}{|c|}{ Maior que $\mathrm{R} \$ 2,4$ milhões e menor ou igual a $\mathrm{R} \$ 16$ milhões } & 14 & $15 \%$ \\
\hline \multicolumn{4}{|c|}{ Maior que $\mathrm{R} \$ 16$ milhões e menor ou igual a $\mathrm{R} \$ 90$ milhões } & 31 & $33 \%$ \\
\hline \multicolumn{4}{|c|}{ Maior que $\mathrm{R} \$ 90$ milhões e menor ou igual a $\mathrm{R} \$ 300$ milhões } & 23 & $25 \%$ \\
\hline \multicolumn{4}{|l|}{ Maior que $\mathrm{R} \$ 300$ milhões } & 15 & $16 \%$ \\
\hline \multicolumn{4}{|l|}{ Total } & 93 & $100 \%$ \\
\hline
\end{tabular}

Fonte: dados da pesquisa.

Na Tabela 2, a atividade econômica que se destaca na amostra investigada é a indústria, que corresponde a $88 \%$. O faturamento médio anual em $33 \%$ das empresas está na faixa entre maior que $\mathrm{R} \$ 16$ milhões e menor ou igual a $\mathrm{R} \$ 90$ milhões, $25 \%$ entre maior que $\mathrm{R} \$ 90$ milhões e menor ou igual a $\mathrm{R} \$ 300$ milhões e $16 \%$ tem faturamento anual acima de $\mathrm{R} \$ 300$ milhões. Isso indica que $74 \%$ dessas empresas são de médio a grande porte, conforme parâmetros do Banco Nacional de Desenvolvimento Econômico e Social (BNDES, 2015). Além disso, 67\% das empresas atuam tanto no mercado nacional quanto no internacional, confirmando que o setor têxtil brasileiro se destaca em âmbito internacional (ABIT, 2017).

Com relação ao tempo de atuação dessas empresas, $61 \%$ têm entre 11 e 50 anos, $29 \%$ atuam há mais de 50 anos no mercado, sendo que, dessas, uma empresa possui 139 anos e apenas $10 \%$ tem até 10 anos de existência. A localização destas empresas concentra-se nas regiões Sul e Sudeste, com 46\% e 39\%, respectivamente. Quanto ao número de funcionários, cerca de 36\% têm entre 100 e 499 funcionários e $39 \%$ mais de 500 funcionários, confirmando que as empresas da amostra são de médio e grande porte.

Quanto aos tipos de cooperação que praticam, 60 empresas indicaram cadeia de suprimentos, 57 terceirização, 26 produção conjunta, 10 franquias e 7 joint ventures. Em média, os respondentes assinalaram que a empresa se envolve em mais de um tipo de cooperação, com destaque para a cadeia de suprimentos e a terceirização, e a menos praticada é a joint venture. Isso indica que as relações entre clientes e fornecedores e a contratação de outra empresa para executar atividades são os tipos mais praticados.

\subsection{Modelo de mensuração}

No modelo de mensuração, avaliaram-se a validade (convergente e discriminante) e a confiabilidade (interna e composta) das medidas dos construtos (Hair Jr, Black, Babin, Anderson \& Tatham, 2014). No modelo original, nenhum construto conseguiu atingir os valores mínimos para a validade convergente (Average Variance Extracted - AVE), acima de 0,5, bem como alguns indicadores apresentaram carga fatorial interna abaixo de 0,4 . Dessa forma, procedeu-se à exclusão dos indicadores até que os construtos atingissem os valores mínimos para a AVE e a carga fatorial interna. Foi necessário realizar três rodadas de testes e exclusões dos indicadores para a validação da confiabilidade dos construtos. 
Após os testes e a exclusão das variáveis que não atingiram os critérios de confiabilidade do modelo, restaram: 10 itens em objetivos de cooperação; 6 em escopo de transação; 6 em práticas de gestão da cooperação; e 13 em desempenho da cooperação (Apêndice A). A Confiabilidade Composta e a AVE do modelo ajustado são apresentadas na Tabela 3. Nota-se que, após as exclusões de itens do modelo teórico, a AVE atingiu os valores mínimos recomendados na literatura (Hair Jr et al., 2014).

Tabela 3

Modelo ajustado

\begin{tabular}{lcl}
\hline \multicolumn{1}{c}{ Construtos } & Confiabilidade Composta & AVE \\
\hline Objetivos da Cooperação & 0,913 & $\mathbf{0 , 5 1 5}$ \\
\hline Escopo de Transação & 0,862 & $\mathbf{0 , 5 1 2}$ \\
\hline Práticas de Gestão da Cooperação & 0,857 & $\mathbf{0 , 5 0 2}$ \\
\hline Desempenho da Cooperação & 0,928 & $\mathbf{0 , 5 0 3}$ \\
\hline
\end{tabular}

Fonte: dados da pesquisa.

As cargas fatoriais estão acima de 0,5 e não há nenhum indicador que esteja altamente correlacionado com outro no modelo ajustado. Na sequência, verificou-se a validade discriminante das variáveis latentes reflexivas de primeira e segunda ordem, que apresenta as correlações entre as variáveis e na diagonal a raiz quadrada da AVE. A correlação entre as variáveis latentes deve ser inferior à raiz quadrada da AVE para se obter a validade discriminante (Fornell \& Larcker, 1981). Na Tabela 4, apresenta-se a validade discriminante do modelo ajustado para as variáveis latentes de primeira e segunda ordem.

Tabela 4

\section{Análise discriminante das variáveis latentes de $1^{\mathrm{a}}$ e $2^{\mathrm{a}}$ ordem}

\begin{tabular}{|c|c|c|c|c|}
\hline Variáveis latentes de $1^{\mathrm{a}}$ ordem & 1 & 2 & 3 & 4 \\
\hline 1. Objetivos da Cooperação & 0,718 & & & \\
\hline 2. Escopo de Transação & 0,578 & 0,715 & & \\
\hline 3. Desempenho da Cooperação & 0,459 & 0,618 & 0,709 & \\
\hline \multicolumn{5}{|l|}{ Variável latente de $2^{\mathrm{a}}$ ordem } \\
\hline 4. Práticas de Gestão da Cooperação & 0,422 & 0,566 & 0,473 & 0,709 \\
\hline Confiabilidade Composta (CC) & & & & 0,812 \\
\hline Variância Média Extraída (AVE) & & & & 0,683 \\
\hline
\end{tabular}

Fonte: dados da pesquisa.

Verifica-se na Tabela 4 que as variáveis latentes do modelo de mensuração têm a raiz quadrada da AVE superior às correlações com as demais variáveis latentes. Após o cálculo da variável latente de segunda ordem, percebe-se que a CC e a AVE estão acima do limite mínimo, confirmando a validade do construto. Os testes de validação do modelo de mensuração permitem analisar o modelo estrutural.

\subsection{Modelo estrutural}

A análise do modelo estrutural foi realizada em duas etapas. Primeiro, analisou-se o $\mathrm{R}^{2}$, que é a porcentagem da variância de uma variável latente dependente que é explicada pelas variáveis independentes (Hair Jr et al., 2014). O bootstraping foi calculado com 5.000 subamostras. Para validar as hipóteses da pesquisa, inicialmente testou-se o modelo estrutural. Para confirmar os resultados encontrados, foi incluída a variável de controle no modelo estrutural. Na Tabela 5, apresentam-se os resultados do modelo estrutural com a variável de controle. 
Tabela 5

\section{Resultado do modelo estrutural}

\begin{tabular}{|c|c|c|c|c|c|c|}
\hline $\begin{array}{c}\text { Relações } \\
\text { estruturais }\end{array}$ & Hipóteses & $\begin{array}{l}\text { Coeficiente } \\
\text { estrutural }\end{array}$ & Valor-t & Valor-p & $F^{2}$ & $\mathbf{R}^{2}$ \\
\hline $\mathrm{OC} \rightarrow \mathrm{DC}$ & $\mathrm{H}_{1}(+)$ & 0,115 & 1,293 & 0,196 & 0,016 & \\
\hline $\mathrm{OC} \rightarrow \mathrm{PC} \rightarrow \mathrm{DC}$ & $\mathrm{H}_{2}(+)$ & 0,092 & 1,986 & $0,047 * *$ & - & 0,424 \\
\hline $\mathrm{OC} \rightarrow \mathrm{ET} \rightarrow \mathrm{DC}$ & $\mathrm{H}_{3}(+)$ & 0,255 & 4,061 & $0,000 * * *$ & - & \\
\hline \multicolumn{7}{|c|}{ Relações Diretas } \\
\hline $\begin{array}{l}\text { Relações } \\
\text { estruturais }\end{array}$ & & $\begin{array}{l}\text { Coeficiente } \\
\text { estrutural }\end{array}$ & Valor-t & Valor-p & $F^{2}$ & $\mathbf{R}^{2}$ \\
\hline $\mathrm{OC} \rightarrow \mathrm{PC}$ & & 0,414 & 3,965 & $0,000 * * *$ & 0,224 & 0,219 \\
\hline $\mathrm{PC} \rightarrow \mathrm{DC}$ & & 0,223 & 2,346 & $0,019 * *$ & 0,056 & 0,424 \\
\hline $\mathrm{OC} \rightarrow \mathrm{ET}$ & & 0,575 & 7,653 & $0,000 * * *$ & 0,500 & 0,324 \\
\hline $\mathrm{ET} \rightarrow \mathrm{DC}$ & & 0,443 & 4,365 & $0,000 * * *$ & 0,192 & 0,424 \\
\hline \multicolumn{7}{|c|}{ Relação Total } \\
\hline $\mathrm{OC} \rightarrow \mathrm{DC}$ & & 0,462 & 5,162 & $0,000 * * *$ & 0,016 & 0,424 \\
\hline \multicolumn{7}{|c|}{ Variáveis de Controle } \\
\hline $\mathrm{TC} \rightarrow \mathrm{PC}$ & & 0,241 & 0,915 & 0,360 & 0,076 & - \\
\hline $\mathrm{TC} \rightarrow \mathrm{ET}$ & & 0,072 & 0,424 & 0,672 & 0,008 & - \\
\hline $\mathrm{TC} \rightarrow \mathrm{DC}$ & & $-0,192$ & 0,979 & 0,328 & 0,062 & \\
\hline
\end{tabular}

Nota: significância ao nível de **5\% e ***1\%.

Legenda: OC = Objetivos da Cooperação; PC = Práticas de Gestão da Cooperação; ET = Escopo de Transação; DC = Desempenho da Cooperação; TC = Tipos de Cooperação.

Fonte: Dados da pesquisa.

De acordo com a Tabela 5, todas as relações têm sinais coerentes com a formulação das hipóteses da pesquisa, ou seja, sinal positivo. A hipótese $\mathrm{H}_{1}$ não encontra suporte para ser aceita estatisticamente. As hipóteses $\mathrm{H}_{2}$ e $\mathrm{H}_{3}$ confirmam mediação. Para avaliar se a mediação é total ou parcial, deve-se verificar os efeitos diretos, indiretos e totais. A relação direta da $\mathrm{H}_{2}$ envolve os objetivos e desempenho da cooperação e não foi estatisticamente significante. A relação indireta entre objetivos e desempenho da cooperação, mediada pelas práticas de gestão da cooperação, foi estatisticamente significante $(\mathrm{p}<0,05)$. O efeito total entre objetivos e desempenho da cooperação apresentou-se estatisticamente significante $(p<0,01)$. Desse modo, a $\mathrm{H}_{2}$ encontra suporte para ser aceita estatisticamente e a mediação é total.

No que concerne à hipótese $\mathrm{H}_{3}$ a relação direta envolve os objetivos e o desempenho da cooperação, mas não se apresentou estatisticamente significante. A relação indireta entre objetivos e desempenho da cooperação, mediada pelo escopo de transação, apresentou-se estatisticamente significante $(\mathrm{p}<0,01)$. $\mathrm{O}$ efeito total entre objetivos e desempenho da cooperação apresentou-se estatisticamente significante $(\mathrm{p}<0,01)$. Dessa forma, aceita-se estatisticamente $\mathrm{a}_{3}$ e a mediação é total.

Quanto à variável de controle, não se encontrou relação estatisticamente significante, o que indica que os tipos de cooperação praticados pelas empresas não influenciam, estatisticamente, os demais elementos da cooperação investigados. No entanto, a inclusão dessa variável no modelo intensificou a significância das relações propostas neste estudo. 


\subsection{Discussão dos resultados}

Os resultados da pesquisa denotam que as hipóteses $\mathrm{H}_{2}$ e $\mathrm{H}_{3}$ formuladas no modelo teórico encontram suporte para serem aceitas estatisticamente. A hipótese $\mathrm{H}_{1}$, que prevê relação positiva entre os objetivos da cooperação e o desempenho da cooperação não foi confirmada. Isso indica que o impacto dos objetivos no desempenho não acontece de forma direta, tendo em vista que as mediações das hipóteses $\mathrm{H}_{2}$ e $\mathrm{H}_{3}$ foram aceitas.

Os objetivos da cooperação são as razões estratégicas para as empresas participarem da cooperação interfirmas (Dekker et al., 2016). Os relacionamentos interfirmas buscam reduzir os custos, acessar novos mercados e tecnologias e maximizar os lucros ou reduzir os riscos (Groot \& Merchant, 2000). Dekker et al. (2016) encontraram relação significativa e positiva entre os objetivos da cooperação e o desempenho da cooperação, e concluíram que a relevância da cooperação está em provocar impactos positivos no desempenho da cooperação, contrariando os resultados encontrados nesta pesquisa.

Nas empresas industriais do setor têxtil pesquisadas, os gestores avaliam a cooperação como estrategicamente importante. No entanto, para que alcancem os objetivos pretendidos com este tipo de aliança estratégica é necessário que as práticas de gestão da cooperação e o escopo de transação estejam alinhados. Para as empresas da amostra, a relevância estratégica da cooperação impacta positivamente o desempenho da cooperação, se estiver associada ao compartilhamento de informações, à interação entre os parceiros e às atividades desenvolvidas entre os parceiros da cooperação, fato que justifica a rejeição da $\mathrm{H}_{1}$.

O aumento da importância estratégica dos objetivos da cooperação é dependente do alinhamento entre os interesses e as ações dos parceiros, o que implica a necessidade de coordenar e controlar as práticas de gestão da cooperação (Reuer, Ariño, 2007; Dekker et al., 2016). De acordo com a importância desses relacionamentos para os parceiros, haverá necessidade de acesso a informações relevantes, o que impacta o aumento do compartilhamento de informações. A demanda por informações exige maior envolvimento dos gestores para gerenciar essas relações, coordenação de ações, reconhecimento das áreas que precisam de melhorias e alinhamento das expectativas, metas e contribuições entre os parceiros (Dekker et al., 2013).

A interpendência entre os interesses dos parceiros, uma das premissas da Teoria da Cooperação (Deustch, 2011), implica que os objetivos da cooperação se relacionem positivamente com as práticas de gestão da cooperação, assim como estas com o desempenho da cooperação. Além de serem influenciadas pelos objetivos da cooperação, as práticas de gestão da cooperação influenciam o desempenho do relacionamento interfirmas. A relação positiva encontrada neste estudo entre objetivos e práticas de gestão da cooperação revela a importância percebida pelos respondentes de que o compartilhamento de informações e a interação entre os parceiros são fundamentais para alcançar o desempenho pretendido, e quanto maior for essa interação, maior será o impacto positivo no relacionamento interfirmas.

Além de geração de receitas e diminuição de custos, a cooperação interfirmas auxilia na troca de conhecimento e informações entre os parceiros (Kogut, 1988). Assim, o gerenciamento da cooperação interfirmas precisa reunir os parceiros pela interação dos expansores de fronteiras (boundary spanners), o que implica o fornecimento de informações que favoreçam decisões alinhadas aos interesses dos parceiros (Mahama, 2006; Dekker et al., 2013). O compartilhamento de informações auxilia os envolvidos na resolução de incertezas, no alcance de metas e no desempenho da cooperação (Mahama, 2006; Dekker et al., 2013).

Neste sentido, as práticas de gestão da cooperação devem estar alinhadas aos objetivos da cooperação por impactarem, positivamente, o desempenho do relacionamento interfirmas (Mahama, 2006; Dekker et al., 2016). O teste da mediação da hipótese $\mathrm{H}_{2}$, que prevê relação positiva entre os objetivos da cooperação e o desempenho da cooperação, mediada pelas práticas de gestão da cooperação, indicou mediação total. 
A importância estratégica da cooperação percebida nas empresas pesquisadas está associada ao maior compartilhamento de informações e interação dos expansores de fronteiras (boundary spanners), maior uso de práticas de gestão da cooperação, o que impulsiona o uso de mecanismos de mensuração do desempenho (Mahama, 2006), que impactam positivamente o desempenho da cooperação. Além disso, quando os objetivos da cooperação são relevantes, como neste estudo, as práticas de cooperação se complementam para diminuir os riscos envolvidos, gerando mais valor aos parceiros (Dekker et al., 2013).

No entanto, o resultado encontrado nesta pesquisa quanto ao compartilhamento de informações diverge dos achados de Mahama (2006). As dimensões de cooperação investigadas pelo autor referemse à resolução conjunta de problemas, vontade de se adaptar às mudanças, restrição do uso de poder e compartilhamento de informações, sendo que, apenas três dimensões da cooperação (resolução de problemas, vontade de se adaptar às mudanças e restrição do uso de poder) mostraram-se associadas diretamente com o desempenho da cooperação. Já a relação entre o compartilhamento de informações e o desempenho da cooperação é indireta. Esse resultado divergente pode ser explicado pela percepção dos gestores das empresas investigadas quanto à importância de compartilhar informações relevantes entre os parceiros para alcançar os objetivos pretendidos.

Além das práticas de gestão da cooperação, outro fator que influencia a relação entre os objetivos e desempenho da cooperação é a extensão com que as atividades desenvolvidas se sobrepõem e geram conhecimentos entre os parceiros, o escopo de transação (Khanna et al., 1998). A hipótese $\mathrm{H}_{3}$, que prevê relação positiva entre os objetivos da cooperação e o desempenho da cooperação, mediada pelo escopo de transação, indicou mediação total. Isso indica que, conforme aumenta a relevância dos objetivos de cooperação e aumenta a inclusão de atividades na cooperação, o escopo torna-se maior (Reuer \& Ariño, 2007). A cooperação com um maior escopo de transação aumenta a possibilidade de extrair benefícios, mas aumenta o risco de exposição do parceiro, o que precisa ser gerenciado (Dekker et al., 2019).

Um escopo de transação maior sinaliza que mais atividades são desenvolvidas na cooperação, e esse envolvimento tende a gerar ganhos financeiros superiores (Kalaignanam et al., 2007). As organizações com escopo de transação maior têm mais benefícios do que as de escopo menor, pois uma cooperação com escopo de transação maior gera mais oportunidades de ganhos (Li et al., 2013). Isso explica as evidências encontradas neste estudo, que quanto maior a extensão das atividades realizadas na cooperação, maior será o efeito entre os objetivos e o desempenho da cooperação.

O efeito da mediação também é confirmado porque o escopo de transação retrata o ambiente interno da cooperação e os setores envolvidos nessa relação que interferem positivamente no alinhamento entre os objetivos e o desempenho das empresas parceiras (Dekker et al., 2016). Isso explica a influência positiva dos objetivos da cooperação no desempenho da cooperação, mediada pelo escopo de transação nas empresas analisadas, o que coadunam com o estudo de Dekker et al. (2016).

O desempenho da cooperação revelou-se positivo, o que sugere que os objetivos dos parceiros possuem interfaces. Desse modo, quando os objetivos dos parceiros estão inter-relacionados, há uma relação de interdependência positiva para o alcance das metas, o que resulta em impacto positivo no desempenho da cooperação conforme previsto na Teoria da Cooperação (Deustch, 2011).

\section{Considerações finais}

Este estudo analisou o efeito das práticas de gestão da cooperação e do escopo de transação na relação entre os objetivos da cooperação e o desempenho da cooperação em empresas da indústria têxtil. Os resultados indicaram que a relação entre os objetivos e o desempenho da cooperação é mediada pelas práticas de gestão da cooperação e pelo escopo de transação. Embora as relações com ambas as mediações tenham sido estatisticamente significantes, o tamanho do efeito das práticas de gestão da cooperação e do escopo de transação em relação ao desempenho da cooperação foi diferente. O tamanho do efeito das práticas de gestão da cooperação foi baixo $(0,056)$, já o escopo de transação foi alto $(0,500)$. 
Desse modo, quanto maior a interação dos envolvidos e o compartilhamento de informações maior será a associação entre os objetivos e o desempenho da cooperação interfirmas nas empresas pesquisadas. Além disso, para os gestores dessas empresas, as atividades desenvolvidas na cooperação podem ter maior relevância do que o compartilhamento de informações e a interação dos expansores de fronteiras (boundary spanners) no desempenho da cooperação interfirmas. Isso se deve ao fato de a maioria dessas empresas praticarem diferentes combinações de formas de cooperação, o que exige que mais operações sejam realizadas nos relacionamentos interfirmas.

Os achados do estudo contribuem para a literatura ao fornecer evidências dos efeitos das práticas de gestão da cooperação e do escopo de transação na relação entre os objetivos e o desempenho das relações interfirmas. Os achados indicaram que o alinhamento entre os objetivos e as práticas de gestão da cooperação interfirmas, assim como o escopo de transação, resultam em melhor desempenho dessas relações. Outra contribuição está na compreensão do fenômeno a partir da Teoria da Cooperação, que está centrada no alinhamento dos objetivos estratégicos entre os participantes das relações interfirmas, o que direciona o esforço dos parceiros para os objetivos mútuos (Mahama, 2006).

A contribuição prática do estudo está na relevância da temática com relação à estratégia adotada pelas organizações para obter vantagens competitivas e proporcionar a criação de valor entre as empresas participantes da cooperação. As organizações têxteis podem promover a cooperação como estratégia para satisfazer às demandas do mercado ao fazerem parte de uma rede inovadora e tecnológica (Bruno, 2016), auxiliando na competitividade estratégica das empresas.

As limitações impostas ao delineamento deste estudo oferecem oportunidades para realizar novas pesquisas sobre a cooperação interfirmas. Estudos futuros podem analisar a cooperação sob a perspectiva aqui analisada em outros contextos, que não o setor têxtil, a fim de comparar os resultados com os dessa pesquisa. Podem também ampliar a análise de outras práticas de gestão da cooperação no relacionamento interfirmas, como sistemas de recompensas e feedback. As relações de cooperação interfirmas também oferecem riscos de transação aos seus parceiros e que podem impactar o desempenho e o sucesso desses relacionamentos. Assim, recomenda-se para pesquisas futuras investigar sobre os riscos de transação e seu impacto na cooperação interfirmas. 


\section{Referências}

Anderson, E. (1990). Two firms, one frontier: On assessing joint venture performance. MIT Sloan Management Review, 31(2), pp. 19-30.

Anderson, S.W., \& Dekker, H.C. (2014). The role of management controls in transforming firm boundaries and sustaining hybrid organizational forms. Foundations and Trends ${ }^{\varpi}$ in Accounting, 8(2), pp. 75141. doi: https://doi.org/10.1561/1400000032

Balestrin, A., Verschoore, J.R., \& Reyes Jr, E. (2010). O campo de estudo sobre redes de cooperação interorganizacional no Brasil. Revista de Administração Contemporânea, 14(3), pp. 458-477. doi: https://doi.org/10.1590/S1415-65552010000300005

Bruno, F.D.S. (2016) A quarta revolução industrial do setor têxtil e de confecção: a visão de futuro para 2030 (1ª Ed.) São Paulo: Estação das Letras e Cores.

Centenaro, A., \& Laimer, C.G. (2017). Relações de cooperação e a competitividade no setor supermercadista. Revista Brasileira de Gestão de Negócios, 19(63), pp. 65-81. doi: https://doi. org/10.7819/rbgn.v0i0.3070

Contractor, F.J., \& Lorange, P. (1988). Why should firms cooperate? The strategy and economics basis for cooperative ventures. In: Contractor, F. J., \& Lorange, P. (eds.). Cooperative strategies in international business 1, pp. 3-30. Lexington, MA: Lexington Books.

Corsten, D., Gruen, T., \& Peyinghaus, M. (2011). The effects of supplier-to-buyer identification on operational performance-An empirical investigation of inter-organizational identification in automotive relationships. Journal of Operations Management, 29(6), 549-560. doi: https://doi. org/10.1016/j.jom.2010.10.002

Das, T.K., \& Teng, B.-S. (1998). Between trust and control: developing confidence in partner cooperation in alliances. Academy of Management Review, 23(3), pp.491-512. doi: https://doi.org/10.5465/ amr.1998.926623

Dekker, H.C. (2016). On the boundaries between intrafirm and interfirm management accounting research. Management Accounting Research, 31(4), pp. 86-99. doi: https://doi.org/10.1016/j. mar.2016.01.001

Dekker, H.C., Ding, R., \& Groot, T. (2016). Collaborative performance management in interfirm relationships. Journal of Management Accounting Research, 28(3), pp. 25-48. https://doi.org/ doi: 10.2308/jmar-51492

Dekker, H., Donada, C., Mothe, C., \& Nogatchewsky, G. (2019). Boundary spanner relational behavior and inter-organizational control in supply chain relationships. Industrial Marketing Management, 77, pp. 143-154. doi: https://doi.org/ 10.1016/j.indmarman.2018.11.010

Dekker, H., Sakaguchi, J., \& Kawai, T. (2013). Beyond the contract: managing risk in supply chain relations. Management Accounting Research, 24(2), pp. 122-139. doi: https://doi.org/ 10.1016/j. mar.2013.04.010

Deustch, M. (2011). Cooperation and competition. In: Deutsch, M. Conflict, interdependence, and justice pp. 23-40. New York: Springer. https://doi.org/10.1007/978-1-4419-9994-8_2

Dias, M.D.C. (2018). Inovação, aprendizagem e cooperação na cadeia de suprimento têxtil da região de Americana/SP. Gestão \& Regionalidade, 34(100), pp.127-148. doi: https://doi.org/ 10.13037/gr.

Ding, R., Dekker, H.C., \& Groot, T. (2013). Risk, partner selection and contractual control in interfirm relationships. Management Accounting Research, 24(2), pp. 140-155. doi: https://doi.org/ 10.1016/j. mar.2013.04.007 
Eiriz, V. (2001). Proposta de tipologia sobre alianças estratégicas. Revista de Administração Contemporânea, 5(2), pp. 65-90. doi: https://doi.org/ 10.1590/S1415-65552001000200004

Faul, F., Erdfelder, E., Buchner, A., \& Lang, A.G. (2009). Statistical power analyses using G*Power 3.1: Tests for correlation and regression analyses. Behavior Research Methods, 41(4), pp. 1149-1160. doi: https://doi.org/10.3758/BRM.41.4.1149

Fornell, C., \& Larcker, D.F. (1981). Structural equation models with unobservable variables and measurement error: algebra and statistics. Journal of Marketing Research, 18 (3), pp. 39-50. doi: https://doi.org/10.1177/002224378101800313

Gibbon, A.R.D.O. (2002). A gestão estratégica de custos de suprimento na perspectiva da cadeia de valor: O caso de uma empresa da indústria têxtil. 2002. 118f. Dissertação de Mestrado, Universidade Federal de Santa Catarina, Florianópolis, SC, Brasil. Recuperado de https://repositorio.ufsc.br/ xmlui/bitstream/handle/123456789/83796/191411.pdf? sequence=1\&isAllowed=y

Grafton, J., Lillis, A.M., \& Widener, S.K. (2010). The role of performance measurement and evaluation in building organizational capabilities and performance. Accounting, Organizations and Society, 35(7), pp. 689-706. doi: https://doi.org/ 10.1016/j.aos.2010.07.004

Groot, T.L., \& Merchant, K.A. (2000). Control of international joint ventures. Accounting, Organizations and Society, 25(6), pp. 579-607. doi: https://doi.org/ 10.1016/S0361-3682(99)00057-4

Hayes, A.F. (2009). Beyond Baron and Kenny: Statistical mediation analysis in the new millennium. Communication Monographs, 76(4), pp. 408-420. doi: https://doi.org/ 10.1080/03637750903310360

Hair Jr, J.F.; Black, W.C.; Babin, B.J.; Anderson, R.E.; Tatham, R.L. (2014). A primer on partial least squares structural equation modeling (PLS-SEM). California: Sage Publications.

Ireland, R.D., Hitt, M.A., \& Vaidyanath, D. (2002). Alliance management as a source of competitive advantage. Journal of Management, 28(3), pp. 413-446. doi: https://doi.org/ 10.1016/S01492063(02)00134-4

Ittner, C.D., Larcker, D.F., Nagar, V., \& Rajan, M.V. (1999). Supplier selection, monitoring practices, and firm performance. Journal of Accounting and Public Policy, 18(3), pp. 253-281. doi: https://doi. org/10.1016/S0278-4254(99)00003-4

Kalaignanam, K., Shankar, V., \& Varadarajan, R. (2007). Asymmetric new product development alliances: win-win or win-lose partnerships? Management Science, 53(3), pp. 357-374. doi: https://doi. org/10.1287/mnsc. 1060.0642

Kaplan, R.S., Norton, D.P., \& Rugelsjoen, B. (2010). Managing alliances with the balanced scorecard. Harvard Business Review, 88(1/2), pp. 114-120. Recuperado de https://hbr.org/2010/01/managing-allianceswith-the-balanced-scorecard

Khanna, T., Gulati, R., \& Nohria, N. (1998). The dynamics of learning alliances: competition, cooperation, and relative scope. Strategic Management Journal, 19(3), pp. 193-210. doi: https://doi.org/10.1002/ (SICI)1097-0266(199803)19:3<193::AID-SMJ949>3.0.CO;2-C

Kogut, B. (1988). A study of the life cycle of joint ventures. Management International Review, 28(4), pp. 39-52. Recuperado de https://www0.gsb.columbia.edu/faculty/bkogut/files/1988_MIR_Kogut.pdf

Langfield-Smith, K., \& Smith, D. (2003). Management control systems and trust in outsourcing relationships. Management Accounting Research, 14(3), 281-307. doi: https://hbr.org/10.1016/S10445005(03)00046-5 
La Rovere, R., Hasenclever, L., Melo, L.M. (2001). Dinâmica da inovação na indústria têxtil e de confecções de Nova Friburgo, RJ. In: Tironi, L. F. (Org.). Industrialização descentralizada: sistemas industriais locais(pp. 338-415. Brasília: Ipea.

Li, P., Tang, G., Okano, H., \& Gao, C. (2013). The characteristics and dynamics of management controls in IJVs: Evidence from a Sino-Japanese case. Management Accounting Research, 24(3), pp. 246-260. doi: https://hbr.org/10.1016/j.mar.2013.04.002

Lunnan, R, \& Haugland, S.A. (2008). Predicting and mea- suring alliance performance: A multidimensional analysis. Strategic Management Journal, 29(5), pp. 545-556. doi: https://hbr.org/10.1002/smj.660

Mahama, H. (2006). Management control systems, cooperation and performance in strategic supply relationships: A survey in the mines. Management Accounting Research, 17(3), pp. 315-339. doi: https://hbr.org/10.1016/j.mar.2006.03.002

May, M.A., \& Doob, L.W. (1937). Competition and cooperation. New York: Social Science Research Council.

Meira, J., Kartalis, N., Tsamenyi, M., \& Cullen, J. (2010). Management controls and inter-firm relationships: a review. Journal of Accounting \& Organizational Change, 6(1), pp. 149-169. doi: https://hbr. org/10.1108/18325911011025731.

Mishra, A., Chandrasekaran, A., \& MacCormack, A. (2015). Collaboration in multi-partner R\&D projects: The impact of partnering scale and scope. Journal of Operations Management, (33/34), pp. 1-14. doi: https://hbr.org/10.1016/j.jom.2014.09.008.

Mouritsen, J., Hansen, A., \& Hansen, C.Ø. (2001). Inter-organizational controls and organizational competencies: episodes around target cost management/functional analysis and open book accounting. Management Accounting Research, 12(2), pp. 221-244. doi: https://hbr.org/10.1006/ mare.2001.0160.

Oxley, J.E., \& Sampson, R.C. (2004). The scope and governance of international R\&D alliances. Strategic Management Journal, 25(8-9), pp. 723-749. doi: https://hbr.org/10.1002/smj.391

Reuer, J.J., \& Ariño, A. (2007). Strategic alliance contracts: dimensions and determinants of contractual complexity. Strategic Management Journal, 28(1), 313-330. doi: https://hbr.org/10.1002/smj.581

Varadarajan, P.R., \& Cunningham, M.H. (1995). Strategic alliances: a synthesis of conceptual foundations. Journal of the Academy of Marketing Science, 23(4), article 282. doi: https://hbr. org/10.1177/009207039502300408

Wilson, K., \& Barbat, V. (2015). The supply chain manager as political-entrepreneur? Industrial Marketing Management, 49(6), pp. 67-79. doi: https://hbr.org/10.1016/j.indmarman.2015.05.034.

Wu, C.W. (2015). Antecedents of franchise strategy and performance. Journal of Business Research, 68(7), pp. 1581-1588. doi: https://hbr.org/10.1016/j.jbusres.2015.01.055 


\section{Apêndice A. Instrumentos de pesquisa}

1. Objetivos da Cooperação (OC) (Dekker et al., 2016)

Indique o quão importantes são os objetivos abaixo para a sua empresa participar de uma cooperação, na escala de 1 (sem importância) a 5 (muito importante).

- OC1.Estabilização de ganhos $\left(^{*}\right)$

- OC2.Compartilhamento de investimentos $\left(^{\star}\right)$

- OC3.Acesso a mão de obra barata $\left(^{*}\right)$

- OC4.Acesso a matérias-primas ou produtos semiacabados $\left(^{*}\right)$

- OC5.Acesso aos mercados locais

- OC6.Redução da concorrência

- OC7.Redução do risco empresarial

- OC8.Acesso à tecnologia e/ou know-how

- OC9.Aprender habilidades de gerenciamento

- OC10.Acesso a uma outra empresa de outro setor industrial

- OC11.Superar barreiras (comércio/investimento)

- OC12.Aumento da capacidade produtiva

- OC13.Aprender as práticas comerciais locais

- OC14.Acesso a redes políticas ou empresariais

Nota: $\left.{ }^{*}\right)$ Itens retirados na análise dos dados.

2. Escopo de Transação (ET) (Ding et al., 2013; Dekker et al., 2016)

Aponte em que medida as operações da cooperação incluem as atividades abaixo, na escala de 1 (pouco) a 5 (muito).

- $\quad$ ET1.Pesquisa e desenvolvimento

- ET2.Engenharia

- ET3.Compras

- ET4.Produção

- ET5.Marketing e vendas

- ET6.Serviço pós-venda $\left(^{*}\right)$

- $\quad$ ET7.Troca de conhecimento

- $\quad$ ET8.Prestação de serviços $\left(^{*}\right)$

Nota: $\left.{ }^{*}\right)$ Itens retirados na análise dos dados.

3. Práticas de Gestão da Cooperação (PC) (Dekker et al., 2016)

\section{Compartilhamento de informações (PC_CI)}

Informe quanta informação sua empresa e seu parceiro trocam sobre os itens abaixo, na escala de 1 (pouca informação) a 5 (muita informação).

- PC1_CI.Custos $\left(^{*}\right)$

- PC2_CI.Vendas

- PC3_CI.Desenvolvimento de produtos/tecnologia $\left(^{*}\right)$

- PC4_CI.Atividades de marketing

- PC5_CI.Performance operacional $\left(^{*}\right)$

- PC6_CI.Recrutamento e treinamento

Nota: $\left.{ }^{*}\right)$ Itens retirados na análise dos dados. 


\section{Interação dos Expansores de Fronteira (Boundary Spanners) (PC_EF)}

Aponte com que frequência os funcionários se reúnem para consultar os abaixo mencionados, na escala de 1 (não consultam) a 5 (periodicamente).

- PC1_EF.A alta administração da cooperação

- PC2_EF.Os gerentes ativos na cooperação

- PC3_EF.Os colaboradores que se dedicam à cooperação

4. Desempenho da Cooperação (DC) (Dekker et al., 2016)

Informe qual a importância dos itens abaixo para avaliar o desempenho da cooperação, na escala de 1 (sem importância) a 5 (muito importante).

- $\quad$ DC1.Lucro $\left(^{*}\right)$

- DC2.Vendas $\left({ }^{*}\right)$

- DC3.Fluxo de caixa

- DC4.Custos $\left(^{*}\right)$

- DC5.Quota de mercado

- DC6.Qualidade de produtos/serviços $\left(^{*}\right)$

- DC7.Satisfação do cliente ${ }^{*}$ )

- DC8.Capacidade utilizada (de produção) $\left(^{*}\right)$

- DC9.Produtividade do trabalho

- DC10.Tempo de entrega $\left(^{*}\right)$

- DC11.Sucesso de transferência de tecnologia

- DC12.Número de introduções de novos produtos

- DC13.Lealdade do colaborador

- DC14.Treinamento de funcionários

- DC15.Número de melhorias para produtos ou serviços

- DC16.Introdução de forma oportuna de produtos

- DC17.Custo por trabalhador

- DC18.Qualidade do serviço pós-venda

- DC19.Melhorias nos sistemas (de produção)

- DC20.Satisfação do empregado

Nota: $\left.{ }^{*}\right)$ Itens retirados na análise dos dados.

5. Tipos de Cooperação (TC) $\left({ }^{\star}\right.$ Eiriz, 2001; ${ }^{*}$ Meira et al., 2010)

Assinale o(s) tipo(s) de cooperação interfirmas que a sua empresa pratica.

- TC1.Cadeia de suprimentos (acordo entre a empresa produtora e seus fornecedores para elaboração do produto destinado ao consumidor final) ${ }^{* *}$.

- TC2.Produção e/ou desenvolvimento de produtos em conjunto (quando duas ou mais empresas produzem conjuntamente os mesmos produtos para atender as necessidades do mercado)*

- TC3.Franquias [processo em que uma empresa (franqueador) concede a outra (franqueado) o direito de explorar uma marca, produto ou técnica de sua propriedade mediante determinadas condições contratuais $]^{* *}$.

- TC4.Terceirização (processo em que uma empresa contrata outra empresa para realizar atividades externas que poderiam ser realizadas internamente pela empresa contratante) ${ }^{*}$.

- TC5.Joint ventures (quando duas ou mais empresas parceiras constituem uma nova empresa para atender um objetivo em comum) $)^{* *}$.

- TC6.Outro. Especifique: 